\title{
Correction to: Embeddability of pairs of weighted quasi-arithmetic means into a semiflow
}

\author{
Dorota GŁazowska, Justyna Jarczyk, and Witold Jarczyk
}

Abstract. A dumb mistake in the formulation of Theorem 6 has been corrected.

Mathematics Subject Classification. Primary 26A18, 26E60, 39B12.

Keywords. Semiflow, Continuous iteration semigroup, Embeddability, Iterability, Iterative root, Weighted quasi-arithmetic mean.

\section{Correction to: Aequat. Math. 94 (2020), 679-687 https://doi.org/10.1007/s00010-019-00674-5}

We made a simple but near-fatal mistake in the statement of Theorem 6 . Namely instead of the assumption " $F(\cdot, t)$ is continuous for each $t \in(0,+\infty)$ " there should be written " $F((x, y), \cdot)$ is continuous for each $(x, y) \in I^{2}$ ". Consequently, the proper formulation of the theorem is as follows:

Theorem 6. Let $F: I^{2} \times(0,+\infty) \rightarrow I^{2}$ be a function such that $F((x, y), \cdot)$ is continuous for each $(x, y) \in I^{2}$. The function $F$ is a continuous semiflow of pairs of weighted quasi-arithmetic means if and only if there exist a function $f \in \mathcal{C} \mathcal{M}(I)$ and numbers $p, q \in(0,1)$ such that $p \geq q$ and

$$
F(\cdot, t)=\left(A_{\mu(t)}^{f}, A_{\nu(t)}^{f}\right), \quad t \in(0,+\infty),
$$

where the functions $\mu, \nu:(0,+\infty) \rightarrow(0,1)$ are given by $(9)$.

The original article can be found online at https://doi.org/10.1007/s00010-019-00674-5. 
Publisher's Note Springer Nature remains neutral with regard to jurisdictional claims in published maps and institutional affiliations.

Dorota Głazowska and Justyna Jarczyk

Faculty of Mathematics, Computer Science and Econometrics

University of Zielona Góra

Szafrana $4 \mathrm{a}$

65-516 Zielona Góra

Poland

e-mail: d.glazowska@wmie.uz.zgora.pl

Justyna Jarczyk

e-mail: j.jarczyk@wmie.uz.zgora.pl

Witold Jarczyk

Institute of Mathematics and Informatics

The John Paul II Catholic University of Lublin

Konstantynów $1 \mathrm{~h}$

20-708 Lublin

Poland

e-mail: w.jarczyk@wmie.uz.zgora.pl 